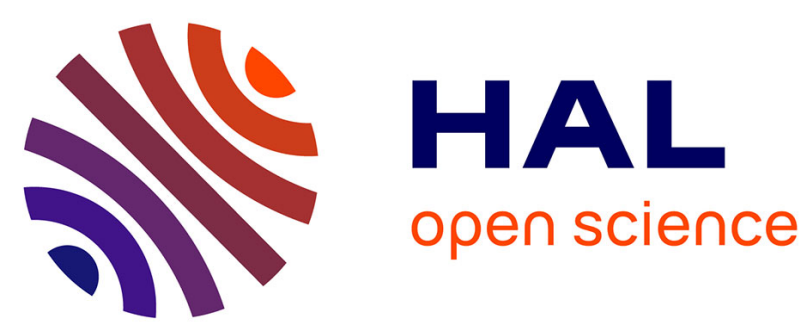

\title{
Contactless Antenna Gain Pattern Estimation From Backscattering Coefficient Measurement Performed Within Reverberation Chambers
}

Ariston Reis, Francois Sarrazin, Philippe Besnier, Philippe Pouliguen, Elodie Richalot

\section{To cite this version:}

Ariston Reis, Francois Sarrazin, Philippe Besnier, Philippe Pouliguen, Elodie Richalot. Contactless Antenna Gain Pattern Estimation From Backscattering Coefficient Measurement Performed Within Reverberation Chambers. IEEE Transactions on Antennas and Propagation, 2022, 70 (3), pp.23182321. 10.1109/TAP.2021.3111184 . hal-03352179

\author{
HAL Id: hal-03352179 \\ https://hal.science/hal-03352179
}

Submitted on 23 Sep 2021

HAL is a multi-disciplinary open access archive for the deposit and dissemination of scientific research documents, whether they are published or not. The documents may come from teaching and research institutions in France or abroad, or from public or private research centers.
L'archive ouverte pluridisciplinaire HAL, est destinée au dépôt et à la diffusion de documents scientifiques de niveau recherche, publiés ou non, émanant des établissements d'enseignement et de recherche français ou étrangers, des laboratoires publics ou privés. 


\title{
Communication Contactless Antenna Gain Pattern Estimation From Backscattering Coefficient Measurement Performed Within Reverberation Chambers
}

\author{
Ariston Reis, François Sarrazin, Member, IEEE, Philippe Besnier, Senior Member, IEEE, Philippe Pouliguen, \\ and Elodie Richalot, Member, IEEE
}

\begin{abstract}
This paper presents the antenna gain pattern estimation from backscattering coefficient measurement performed within a reverberation chamber $(\mathrm{RC})$. The antenna backscattering coefficient is measured for two load conditions, namely an open circuit and a $50 \Omega$ load, enabling to distinguish the radiation and structural modes from the total backscattered field. This approach is applied to the measurement of the gain pattern of a Vivaldi antenna at $6.5 \mathrm{GHz}$ and compared to a conventional transmission-type measurement within an anechoic chamber. The main lobe of the radiation pattern is in good agreement between both measurements. The difference between maximal gains, retrieved after a reference measurement using a metallic plate, is equal to $0.04 \mathrm{~dB}$. A second validation is performed using a log-periodic antenna at $3.5 \mathrm{GHz}$, showing less than $0.76 \mathrm{~dB}$ difference over the measured angular range. To the author's knowledge, this is the first time that a contactless gain pattern measurement is performed and validated within an RC.
\end{abstract}

Index Terms-Reverberation chamber, radar cross-section, antenna gain, antenna backscattering coefficient, structural mode, radiation mode.

\section{INTRODUCTION}

The gain as well as the radiation pattern are crucial antenna properties usually evaluated through transmission-type measurements. This measurement implies an invasive setup with excitation cables connected to the antenna under test (AUT). In the case of electrically small antennas, the presence of cables in the antenna near-field zone can disturb both the impedance and radiation properties and lead to inaccurate results. The literature reports two main paths of investigation to overcome this issue: the first one consists in either limiting the cables effect by adding ferrites, quarter-wavelength sleeves or balun [1], or compensating it thanks to prior electromagnetic simulations [2], whereas the second one aims at avoiding the use of these excitation cables connected to the AUT. Inspired from radar cross-section (RCS) measurement, such method relies on the antenna backscattering characterization to retrieve its radiation properties in a contactless or non-invasive way.

The antenna scattering properties have been first investigated by King in 1949 [3] and methodologies to retrieve

Submitted February 24, 2021; revised August XX,2021. This work was supported in part by the Direction Générale de l'Armement (DGA) of the French Ministry of the Armed Forces.

A. Reis, F. Sarrazin and E. Richalot are with the ESYCOM lab, Univ Gustave Eiffel, UMR CNRS 9007, F-77454 Marne-la-Vallée, France (e-mail: francois.sarrazin@univ-eiffel.fr).

P. Besnier is with Univ Rennes, INSA Rennes, CNRS, IETR-UMR 6164, F-35000 Rennes, France (e-mail: Philippe.Besnier@insa-rennes.fr).

P. Pouliguen is with the Defence Innovation Agency, French Ministry of the Armed Forces.

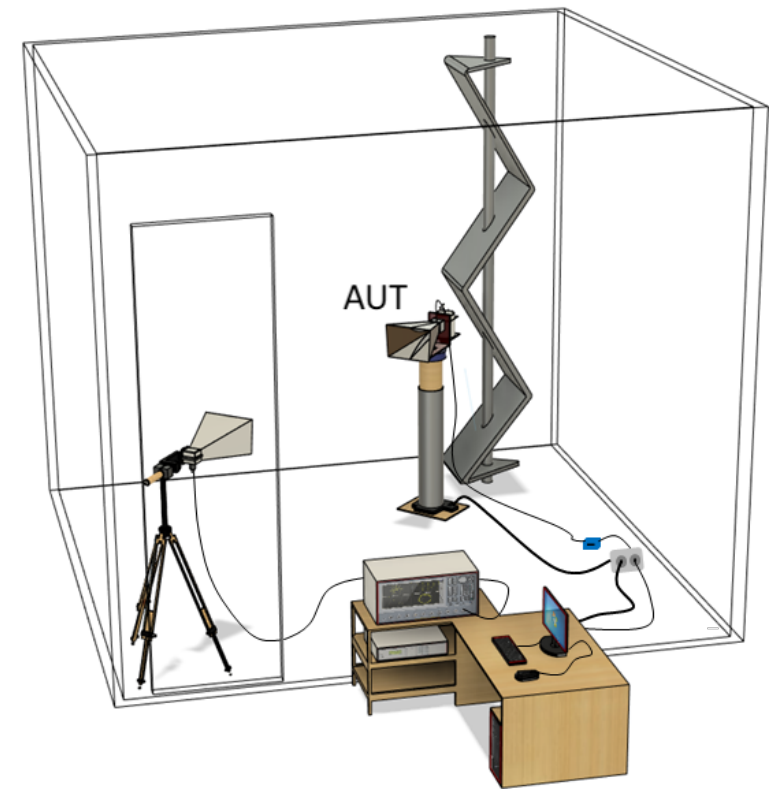

Fig. 1. Measurement setup within the RC. The AUT is located on a mast rotating over the azimuthal angle and is connected to a mechanical switch.

antenna parameters have been continuously of interest since then [4]-[6]. The field backscattered by an antenna consists of two components: the structural mode, due to the antenna seen as a simple object, and the radiation mode, also called the antenna mode, due to the received field transmitted towards the port of the antenna and then reradiated according to impedance mismatches. Solely the radiation mode needs to be accounted for to retrieve the antenna radiation properties. Methods to distinguish between the two modes are based on a load variation approach, either using length-varying transmission line [5] or discrete load impedances [6].

RCS measurements are traditionally performed within an anechoic chamber (AC) that mimics a free-space environment. However, reverberation chambers (RCs) recently became a reliable alternative to ACs for RCS measurements. Two approaches have been investigated: one relies on the line-ofsight path contribution selection through a time-gating postprocess [7], whereas the other one takes benefit from the diffuse-field properties within the RC. The original idea has been introduced in [8] for a fixed stirrer position whereas the RCS accuracy improvement through the stirring process has been highlighted in [9]-[11]. However, up to now, all RCS measurements performed within RCs have only considered 
simple metallic targets such as a plate or a dihedral. In this paper, we consider the specific case of an antenna whose RCS is composed of the two aforementioned modes. More specifically, the objective of this paper is to demonstrate for the first time the possibility to estimate the antenna gain and radiation pattern from antenna backscattering coefficient measurements performed within RCs. This paper is organized as follows. Section II presents the theory to extract the antenna gain from the backscattering coefficient measured within an RC. The experimental setup as well as the measurement of a Vivaldi antenna are presented in Section III where the gain pattern results are compared to the one measured in an AC. A second validation using a log-periodic antenna is performed in Section IV. Finally, a conclusion ends this paper.

\section{THEORY}

\section{A. Antenna Backscattering Coefficient Within an RC}

The monostatic measurement setup within an RC is presented in Fig. 1. A measurement antenna, connected to a VNA, is oriented towards the AUT located on a mast rotating over the azimuthal plane according to the $\theta$ angle. The two antennas are separated by a distance $R$. The scattering parameter $S\left(f, \alpha, \theta, \Gamma_{\mathrm{L}}\right)$ measured by the measurement antenna at the frequency $f$ and for the stirrer angular position $\alpha$, when the AUT is terminated with a load impedance leading to the reflection coefficient $\Gamma_{\mathrm{L}}$, can be expressed as:

$$
\begin{array}{r}
S\left(f, \alpha, \theta, \Gamma_{\mathrm{L}}\right)=S_{\mathrm{FS}}(f)+ \\
\left(1-\left|S_{\mathrm{FS}}(f)\right|^{2}\right) \eta \times\left[H\left(f, \alpha, \theta, \Gamma_{\mathrm{L}}\right)+h_{\mathrm{S}}(f, \alpha)\right]+ \\
C(f) \times \sqrt{\sigma\left(f, \theta, \Gamma_{\mathrm{L}}\right)}
\end{array}
$$

where $S_{\mathrm{FS}}(f)$ and $\eta$ are the free-space reflection coefficient and the radiation efficiency of the measurement antenna, respectively, $H\left(f, \alpha, \theta, \Gamma_{\mathrm{L}}\right)$ is the complex-valued $\mathrm{RC}$ transfer function describing the backscattered signal towards the antenna associated to the diffuse field (related to multiple reflections and diffractions within the cavity), $h_{\mathrm{s}}(f, \alpha)$ accounts for line-of-sight or specular reflections from the $\mathrm{RC}$ walls and mechanical stirrer towards the measurement antenna. The term $C(f) \times \sqrt{\sigma\left(f, \theta, \Gamma_{\mathrm{L}}\right)}$ corresponds to the ballistic wave backscattered by the AUT, with $\sqrt{\sigma\left(f, \theta, \Gamma_{\mathrm{L}}\right)}$ the AUT backscattering coefficient in which the phase information is retained [12], and the factor $C(f)$ can be evaluated from the radar equation as:

$$
C(f)=\frac{G(f) \lambda\left(1-\left|S_{\mathrm{FS}}(f)\right|^{2}\right)}{(4 \pi)^{3 / 2} R^{2}} \exp \left(-\mathrm{j} 2 \pi f \frac{2 R}{c}\right)
$$

where $G(f)$ is the measurement antenna gain, $\lambda$ is the wavelength, and $c$ is the speed of light. According to the antenna scattering theory, the antenna backscattering coefficient $\sqrt{\sigma\left(f, \theta, \Gamma_{\mathrm{L}}\right)}$ can be split into two parts. The first one $\sqrt{\sigma_{\mathrm{s}}(f, \theta)}$ is called the structural mode and is related to the wave backscattered by the AUT as an object, independently of its radiation properties. The second one $\sqrt{\sigma_{\mathrm{r}}\left(f, \theta, \Gamma_{\mathrm{L}}\right)}$ is called the radiation mode and is due to the wave received by the antenna at its receiving port and reradiated according to the reflection coefficient $\Gamma_{\mathrm{L}}$. The antenna backscattering coefficient can thus be written as:

$$
\sqrt{\sigma\left(f, \theta, \Gamma_{\mathrm{L}}\right)}=\sqrt{\sigma_{\mathrm{s}}(f, \theta)}+\sqrt{\sigma_{\mathrm{r}}\left(f, \theta, \Gamma_{\mathrm{L}}\right)} .
$$

The structural mode does not depend on $\Gamma_{\mathrm{L}}$ whereas $\sqrt{\sigma_{\mathrm{r}}\left(f, \theta, \Gamma_{\mathrm{L}}\right)}$ can be expressed as:

$$
\sqrt{\sigma_{\mathrm{r}}\left(f, \theta, \Gamma_{\mathrm{L}}\right)}=\sqrt{\sigma_{\mathrm{r}}^{\max }(f, \theta)} \times \Gamma_{\mathrm{L}}(f)
$$

where $\sqrt{\sigma_{\mathrm{r}}^{\max }(f, \theta)}$ is the antenna backscattering coefficient due to the radiation mode for $\Gamma_{\mathrm{L}}=1$.

\section{B. Antenna Gain Extraction}

Solely the radiation mode should be considered in order to retrieve antenna radiation characteristics. In order to separate it from the structural component, we consider two measurements performed for two different load conditions $\Gamma_{\mathrm{L} 1}(f)$ and $\Gamma_{\mathrm{L} 2}(f)$. The difference between the two measured $\mathrm{S}$ parameters can be expressed as:

$$
\begin{aligned}
& S\left(f, \alpha, \theta, \Gamma_{\mathrm{L} 1}\right)-S\left(f, \alpha, \theta, \Gamma_{\mathrm{L} 2}\right)= \\
& C(f) \times\left(\sqrt{\sigma\left(f, \theta, \Gamma_{\mathrm{L} 1}\right)}-\sqrt{\sigma\left(f, \theta, \Gamma_{\mathrm{L} 2}\right)}\right)+n(f, \alpha, \theta)
\end{aligned}
$$

with

$$
\begin{aligned}
& n(f, \alpha, \theta)= \\
& \left(1-\left|S_{\mathrm{FS}}(f)\right|^{2}\right) \eta \times\left[H\left(f, \alpha, \theta, \Gamma_{\mathrm{L} 1}\right)-H\left(f, \alpha, \theta, \Gamma_{\mathrm{L} 2}\right)\right] .
\end{aligned}
$$

Putting (3) and (4) into (5), it comes:

$$
\begin{aligned}
& S\left(f, \alpha, \theta, \Gamma_{\mathrm{L} 1}\right)-S\left(f, \alpha, \theta, \Gamma_{\mathrm{L} 2}\right)=C(f) \times \\
& \sqrt{\sigma_{\mathrm{r}}^{\max }(f, \theta)} \times\left(\Gamma_{\mathrm{L} 1}(f)-\Gamma_{\mathrm{L} 2}(f)\right)+n(f, \alpha, \theta) .
\end{aligned}
$$

This difference is composed of two terms: the first one is proportional to the AUT backscattering coefficient related to its radiation mode. Indeed, the structural mode being the same for both load conditions, it vanishes. We notice from (2) that this term behaves as a sine wave signal as a function of the frequency with a $c / 2 R$ period. The second term $n(f, \alpha, \theta)$ is proportional to the difference of two random variables whose real and imaginary parts are distributed according to a centered Gaussian probability density function with equal variance. This second term can be seen as an interfering signal that is summed to the wave backscattered by the AUT.

As already shown in our previous studies of target RCS retrieval from measurement in an RC [9]-[11], the stirring process permits to drastically reduce the amplitude of this interfering signal and thus improve the extraction accuracy of the signal of interest. To this end, an average of (7) is performed over a stirrer rotation. Besides, to compensate the variation over the frequency range of the measurement antenna properties along with the wavelength and the load reflection coefficients that impacts the sine wave amplitude according to (2) and (7), the term $\sqrt{\sigma_{\mathrm{r}}^{\max }(f, \theta)}$ is extracted using a 


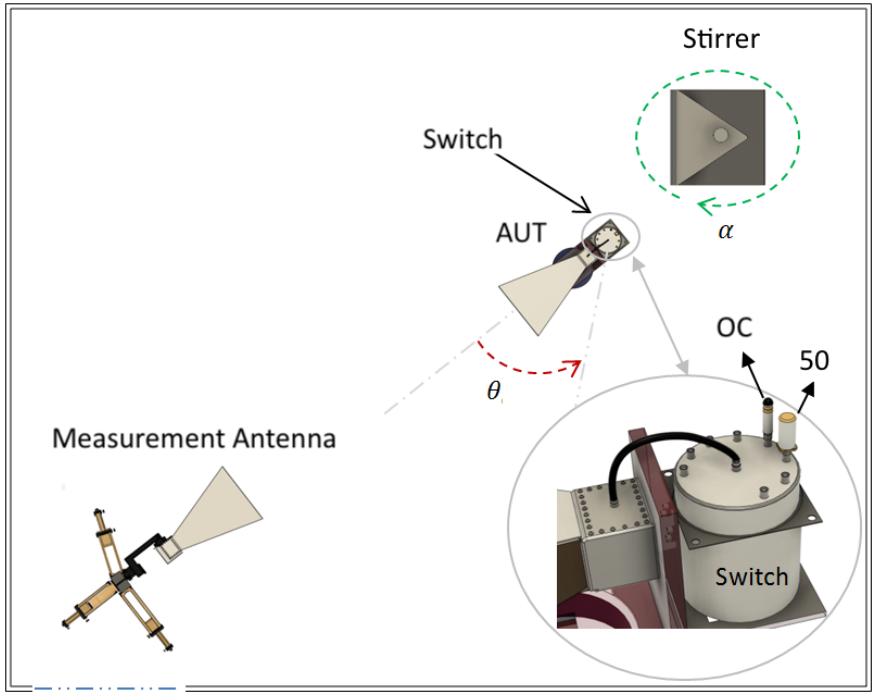

Fig. 2. Contactless RC measurement setup from the top view. Zoom on the switch connection to the AUT and the loads.

regression process from the corrected difference $\Delta_{S}$ in (8) (see [10] for full details).

$$
\begin{aligned}
& \Delta_{S}\left(f, \theta, \Gamma_{\mathrm{L} 1}, \Gamma_{\mathrm{L} 2}\right)= \\
& \frac{\left\langle S\left(f, \alpha, \theta, \Gamma_{\mathrm{L} 1}\right)-S\left(f, \alpha, \theta, \Gamma_{\mathrm{L} 2}\right)\right\rangle_{\alpha}}{G(f) \lambda\left(1-\left|S_{\mathrm{FS}}(f)\right|^{2}\right) \times\left(\Gamma_{\mathrm{L} 1}(f)-\Gamma_{\mathrm{L} 2}(f)\right)}
\end{aligned}
$$

where $\langle.\rangle_{\alpha}$ indicates an average over all stirrer positions $\alpha$. Once $\sqrt{\sigma_{\mathrm{r}}^{\max }(f, \theta)}$ is known, the AUT gain $G_{\mathrm{AUT}}(f, \theta)$ can be computed as [12]:

$$
G_{\mathrm{AUT}}(f, \theta)=\frac{\sqrt{4 \pi}}{\lambda} \times \sqrt{\left|\sigma_{\mathrm{r}}^{\max }(f, \theta)\right|} .
$$

\section{Vivaldi Antenna Measurement}

\section{A. Experiment}

All experiments have been conducted in the ESYCOM RC whose dimensions are $2.95 \times 2.75 \times 2.55 \mathrm{~m}^{3}$. Its lowest usable frequency is estimated about $400 \mathrm{MHz}$ and it is equipped with a metallic mechanical mode stirrer. The setup is presented in Fig. 2. The measurement antenna is a directive horn antenna whose S-parameters are measured in the 6 to $7 \mathrm{GHz}$ frequency range using 20001 frequency points and 72 equally spaced stirrer positions. The AUT is a Vivaldi antenna that rotates in the azimuthal plane from $\theta=-90^{\circ}$ to $\theta=90^{\circ}$ with a $1^{\circ}$ degree step, $\theta=0^{\circ}$ corresponding to the boresight direction. The two antennas are separated by a distance $R=2.29 \mathrm{~m}$. In order to maximize the reflection coefficient difference and thus enhance the measurement sensitivity, we chose an open circuit (OC) and a $50 \Omega$ load for the two considered load impedances. A $30-\mathrm{cm}$ coaxial cable connects the AUT to a mechanical switch terminated by the two loads. The reflection coefficient $\Gamma_{\mathrm{L}}$ at the antenna access due to the mismatch between the AUT and the load impedance is deduced from the measurement of

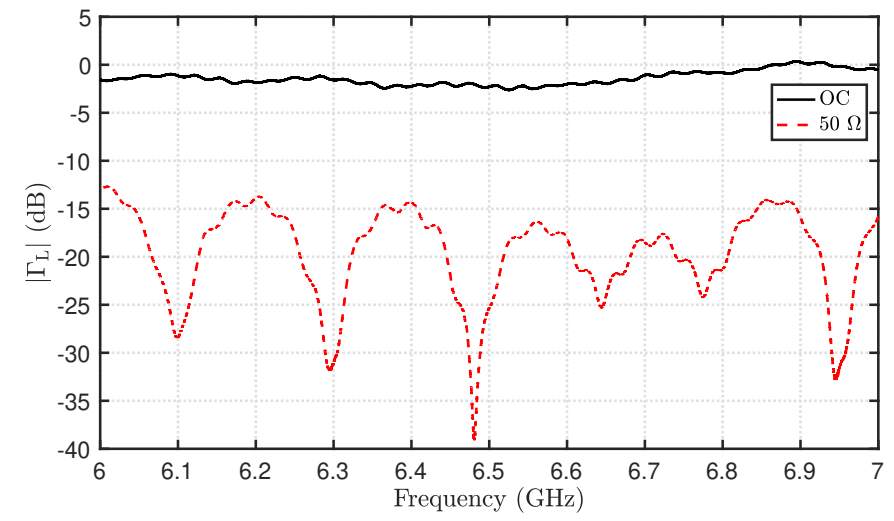

Fig. 3. Reflection coefficient $\left|\Gamma_{L}\right|$ in $\mathrm{dB}$ as a function of frequency for the $\mathrm{OC}$ and $50 \Omega$ loads.

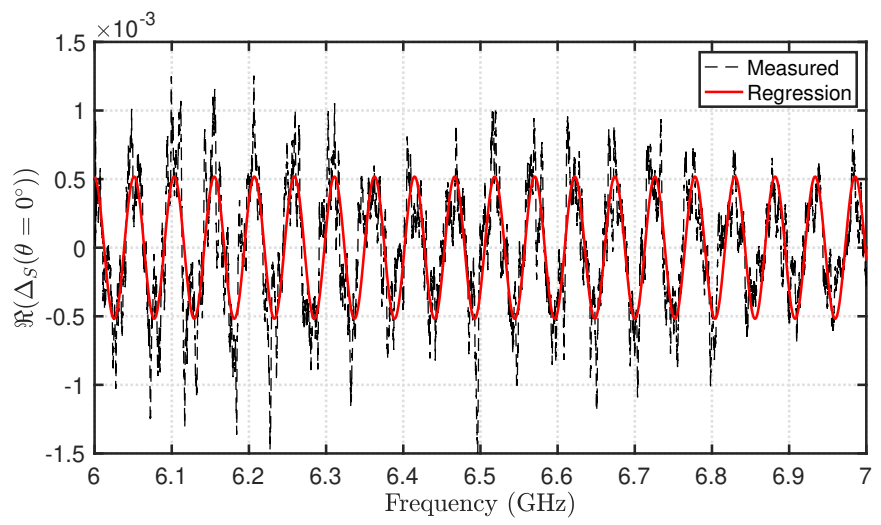

Fig. 4. Real part of $\Delta_{S}$ for AUT azimutal angle $\theta=0^{\circ}$ averaged over all stirrer positions as a function of frequency.

the reflection coefficient between the AUT and $50 \Omega\left(\Gamma_{\mathrm{a}}\right)$ and the one between the load and $50 \Omega\left(\Gamma_{l}\right)$ from

$$
\Gamma_{\mathrm{L}}(f)=\frac{1-\Gamma_{\mathrm{a}}(f)}{1+\Gamma_{\mathrm{a}}^{\dagger}(f)} \times \frac{\Gamma_{l}(f)-\Gamma_{\mathrm{a}}^{\dagger}(f)}{1-\Gamma_{\mathrm{a}}(f) \Gamma_{l}(f)} .
$$

where the superscript $\dagger$ stands for the complex conjugate. The Fig. 3 presents $\left|\Gamma_{\mathrm{L}}\right|$ for the OC and $50 \Omega$ loads computed using (10). The OC case exhibits losses of about $1.5 \mathrm{~dB}$ on average over the frequency band; these are mainly due to the switch insertion losses. For the $50 \Omega$ load, $\left|\Gamma_{L}\right|$ remains lower than $-13 \mathrm{~dB}$ on the entire frequency range.

The Fig. 4 presents the real part of $\Delta_{S}$ for the AUT azimuthal angle $\theta=0^{\circ}$ averaged over all stirrer positions as a function of frequency (dashed black curve). The expected sine wave behavior can be observed as a function of frequency as well as the presence of additive noise due to the $\mathrm{RC}$ reflections. The amplitude of the sine function for all AUT orientations $\theta$ is extracted through a regression process. The retrieved sine wave for $\theta=0^{\circ}$ is plotted in Fig. 4 (solid red curve). The frequency period is about $52 \mathrm{MHz}$ that is equivalent to a distance of $2.89 \mathrm{~m}$. This is consistent with the actual distance $\mathrm{R}$ between antennas once the length from the antenna aperture to the calibration reference plane for both antennas has been added. Calibration is performed thanks to the RCS measurement of a $148 \times 151 \mathrm{~mm}^{2}$ metallic plate in the broadside incidence, using 


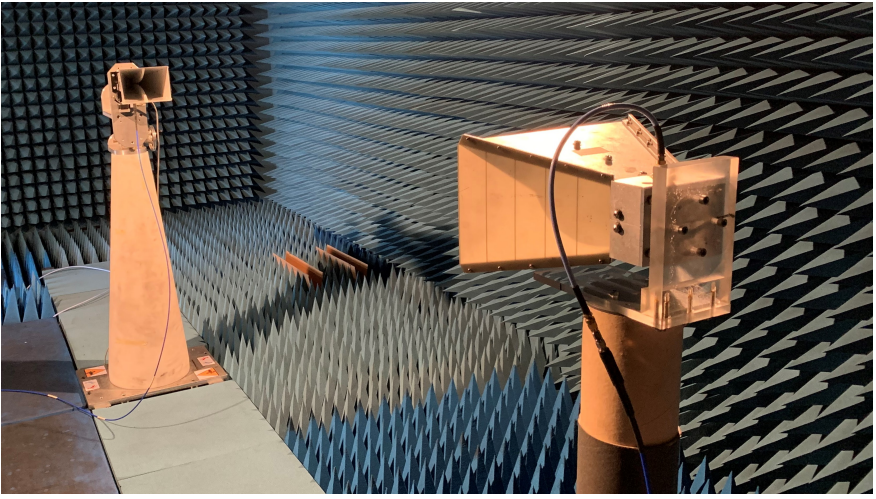

Fig. 5. Transmission-type measurement setup within anechoic chamber. The distance between antennas is identical to that of RC.

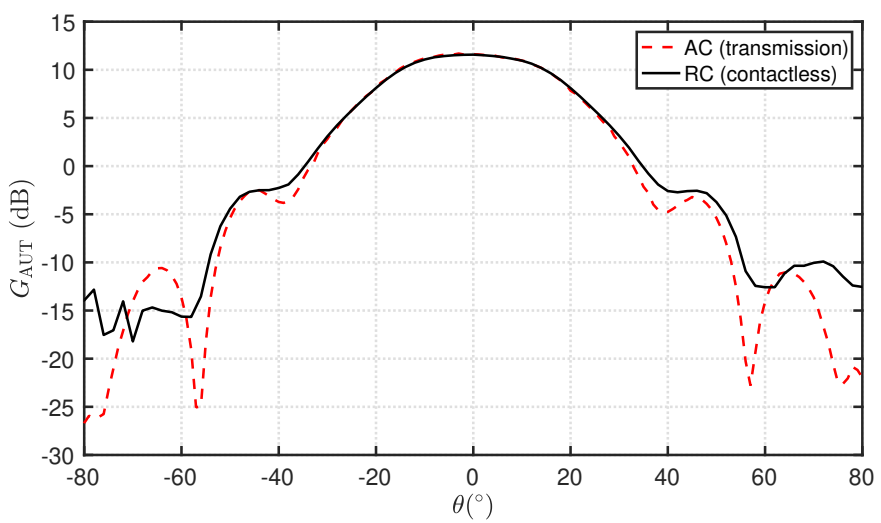

Fig. 6. Measured gain pattern of the Vivaldi antenna at $6.5 \mathrm{GHz}$ as a function of the azimuthal angle $\theta$.

the exact same experimental setup within the RC. The AUT gain $G_{\mathrm{AUT}}(f, \theta)$ can then be extracted from (9). To provide a comparison, the antenna gain pattern is measured within an $\mathrm{AC}$ by the conventional transmission approach whose setup is presented in Fig. 5, for the same distance $R$ between antennas and in the same azimuthal plane. A double ridged horn antenna (Schwarzbeck BBHA 9120 B) of known gain has been used for calibration in order to extract the gain absolute value from this measurement.

\section{B. Results}

The gain pattern measured at $6.5 \mathrm{GHz}$ by the contactless method in the RC is presented in Fig. 6 and compared with the one measured using the conventional transmission setup in $\mathrm{AC}$. The boresight gain is very similar: $11.57 \mathrm{~dB}$ with the $\mathrm{RC}$ contactless approach versus $11.61 \mathrm{~dB}$ with the $\mathrm{AC}$ setup, as well as the half power beamwidth: $37.8^{\circ}$ with the $\mathrm{RC}$ contactless approach versus $37.9^{\circ}$ with the AC setup. The main lobe is in good agreement between both measurements, especially when $|\theta|<30^{\circ}$ (less than $0.70 \mathrm{~dB}$ difference) whereas the side lobes after $|\theta|=50^{\circ}$ are not well retrieved using the contactless RC setup due to the very low signal to interference ratio. This ratio could be enhanced by using other types of mode stirring.

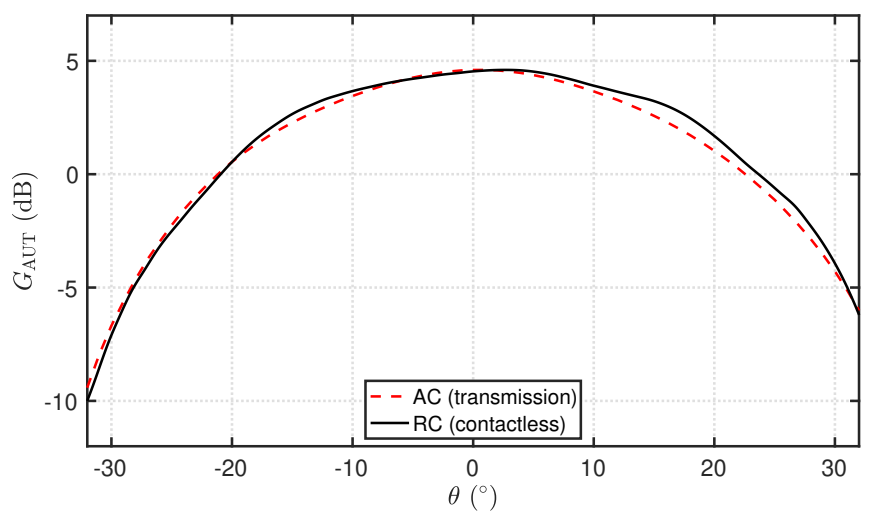

Fig. 7. Measured gain pattern of the log-periodic antenna at $3.5 \mathrm{GHz}$ as a function of the angle $\theta$.

\section{LOG-PERIODIC Antenna MeAsurement}

In this section, we validate the introduced gain pattern measurement method on a second antenna, namely a logperiodic antenna (Schwarzbeck VUSLP 9111-400), in the 3 to $4 \mathrm{GHz}$ frequency range using the same setup than the previous measurement. Due to practical limitations regarding this antenna support, the angular range is limited to $\theta=-32^{\circ}$ to $\theta=32^{\circ}$ with a $1^{\circ}$ degree step. As for the previous measurement, a $1 \mathrm{GHz}$ frequency window is selected around the central frequency to perform the sinusoidal regression. The log-periodic gain pattern is presented in Fig. 7, compared to the transmission-type AC measurement at $3.5 \mathrm{GHz}$. Both results are in good agreement and exhibit, for $|\theta|<30^{\circ}$, a maximum difference of $0.76 \mathrm{~dB}$ and an average absolute difference of $0.31 \mathrm{~dB}$. Measurements have been normalized by the maximum gain given by the datasheet as no calibration measurement has been performed for this study.

\section{CONCLUSION}

In this paper, the antenna gain pattern has been evaluated for the first time from the backscattering coefficient measured within an RC. The radiation mode of the AUT backscattering coefficient has been extracted thanks to measurements performed for two load conditions, namely an OC and a $50 \Omega$ load. It has been validated in a $19 \mathrm{~m}^{3} \mathrm{RC}$ using a Vivaldi antenna in the 6 to $7 \mathrm{GHz}$ frequency range and a log-periodic antenna in the 3 to $4 \mathrm{GHz}$ frequency range. The main lobe for all measurements is in good agreement with traditional transmission-type measurement performed within an $\mathrm{AC}$ (maximum difference of $0.70 \mathrm{~dB}$ for the Vivaldi antenna and $0.76 \mathrm{~dB}$ for the log-periodic antenna). Also, the maximum gain is very well retrieved $(0.04 \mathrm{~dB}$ difference for the Vivaldi antenna) thanks to a calibration using a metallic plate.

\section{REFERENCES}

[1] C. Icheln, J. Ollikainen, and P. Vainikainen, Reducing the influence of feed cables on small antenna measurements, Electron. Lett., vol. 35, no. 15, pp. 1212-1214, July 1999.

[2] L. Huitema, C. Delaveaud and R. D'Errico, Impedance and radiation measurement methodology for ultra miniature antennas, IEEE Trans. Antennas Propag., vol. 62, no. 7, pp. 3463-3473, July 2014, doi: 10.1109/TAP.2014.2320534. 
[3] D. King, The measurement and interpretation of antenna scattering, Proc. IRE, vol. 37, no. 7, pp. 770 - 777, July 1949.

[4] R. J. Garbacz, Determination of antenna parameters by scattering crosssection measurements, Proc. Inst. Elect. Eng., vol. 111, no. 10, pp. 1679-1686, Oct. 1964.

[5] J. Happel-Hansen, Accurate determination of gain and radiation patterns by radar cross-section measurements, IEEE Trans. Antennas Propag, vol. 27, no. 5, pp 640-646, 1979, doi: 10.1109/TAP.1979.1142156.

[6] W. Wiesbeck and E. Heidrich, Wide-band multiport antenna characterization by polarimetric RCS measurements, IEEE Trans. Antennas Propag, vol. 46, no. 3, pp 341-350, Mar. 1998, doi: 10.1109/8.662653.

[7] A. Soltane, G. Andrieu and A. Reineix, Monostatic Radar cross-section estimation of canonical targets in reverberating room using time-gating technique, Int. Symp. Electromagn. Compat., Aug. 2018, pp. 355-359, doi: 10.1109/EMCEurope.2018.8485084.

[8] P. Besnier, J. Sol, S. Méric, Estimating radar cross-section of canonical targets in reverberation chamber, 2017 Int. Symp. Electromagn. Compat., Sep. 2017, pp 1-5, doi: 10.1109/EMCEurope.2017.8094795.

[9] A. Reis, F. Sarrazin, E. Richalot and P. Pouliguen, Mode-stirring impact in radar cross section evaluation in reverberation chamber, 2018 Int. Symp. Electromagn. Compat., Aug. 2018, pp 875-878, doi: 10.1109/EMCEurope.2018.8485023.

[10] A. Reis, F. Sarrazin, E. Richalot, S. Méric, J. Sol, P. Pouliguen and $\mathrm{P}$. Besnier, Radar cross-section pattern measurements in a modestirred reverberation chamber: theory and experiments, IEEE Trans. Antennas Propag., vol. 69, no. 9, pp. 5942-5952, Feb. 2021, doi: 10.1109/TAP.2021.3060581.

[11] A. Reis, F. Sarrazin, P. Pouliguen, J. Sol, P. Besnier and E. Richalot, Radar cross section measurement within reverberation chamber: stirrer position issues, Europ. Conf. Antennas Propag., Mar. 2020, pp 1-4, doi:10.23919/EuCAP48036.2020.9135531.

[12] E. F. Knott, J.F. Shaeffer, M. T. Tuley, Radar Cross Section, $2^{\text {nd }}$ edition, SciTech Publishing, 2004, pp. 19, 409-410. 